\section{Compression treatment following polytrauma and in endocrinopathies}

\section{Mieke Flour}

Phlebology and Chronic Wounds Clinic, Vascular Centre, University Hospital, St. Raphaël (KUV), Leuven, Belgium

Management of polytrauma patients will aim as much as possible at salvation of limbs and limb function if possible. The same applies to surgical reconstruction, for example of deglovement injury. Limb saving flaps and grafts may lead to complex loco-regional situations, with non-homogenic hardness or elasticity, fragility of tissues and dysfunctional skin. Inevitable damage to nerves, blood vessels or lymphatics prolongs healing or results in stiff scarred parts while other sites may be swollen (Figure 1). Sometimes, flaps and grafts appear to be undermined due to difficulties concerning adherence to the underlying tissues. Scarring may further impair elasticity or skin and limb functions. Following closure or healing of skin defects, rehabilitation exercises must start without compromising the fragile skin and eventually orthoses/prostheses will have to be accommodated. Therefore, compression treatment is sometimes needed to reshape or to protect the limb. The mobility and the need for compression may not be equal in the different compartments of the limb to be treated, and often compression devices will have to be adapted towards a combination of different materials and techniques, in order to deliver appropriate local pressure values. If the shape of the post-traumatic limb is too difficult to accommodate with standard devices, a silicone orthosis might help to reshape the leg in order to permit bandaging or donning of a compression stocking (Figure 2). At the same time this orthosis will equalize consistency of tissues and protect utterly fragile parts.

The endocrinopathies considered in relation to compression therapy are mainly diabetes, thyroid disorders, morbid obesity and the metabolic syndrome, as they induce skin changes prone to collect and retain edema fluid, as well as vascular changes leading to hypertension and endothelial dysfunction. In peripheral arterial insufficiency, such as in hypertension, diabetes, peripheral arterial occlusive disease, it is important to keep in mind the territories of the affected angiosomes in order to adapt pressures accordingly.

Compression affects the venous, arterial, lymphatic and capillary circulation. Not only the mechanical effects but also metabolic and behavioral changes on the cellular level have been described for endothelial cells, circulating leukocytes, or fibroblasts on the level of the microcirculation, being it primarily in phlebological patients. It has been shown that in patients with mixed disease [ankle-brachial index (ABI) 0.5-0.8] compression enhances arterial circulation by removal of edema through reduction in capillary filtration, increasing lymphatic drainage, a shift of fluid to non-compressed areas, and a breakdown of fibrosclerotic tissue (due to inflammatory mechanisms in venous disease).
Correspondence: Mieke Flour, Schoonzichtlaan 43, B-3020 Herent, Belgium. Tel. +32.478 .566780 . E-mail: mie.flour@skynet.be

This work is licensed under a Creative Commons Attribution 4.0 License (by-nc 4.0).

CC Copyright M. Flour, 2016

Licensee PAGEPress, Italy

Veins and Lymphatics 2016; 5:5983

doi:10.4081/vl.2016.5983

Endothelial dysfunction is strongly associated with both type- 1 and -2 diabetes mellitus.

In diabetic patients, the attention is cautiously focused on the micro-and macroangiopathy and on a peripheral sensory neuropathy considering the failure of perception under inappropriately high-pressure values. Multiple abnormalities in the skin microcirculation of the foot have been described in diabetics with neuropathy, and controversial results depending on the methods used are sometimes difficult to interpret. ${ }^{1}$ Peripheral autonomic neuropathy impacts on the regulation of flow in the small vessels of the skin including the lymphatics.

These subjects may present edema due to several organ complications like heart or kidney failure, swelling secondary to the medication to treat these, or caused by the diabetic tissue alterations of the skin. Advanced glycation endproducts (and lipoxigenation), celland matrix alterations as well as deposition of a.o. amyloid [and glycosaminoglycans (GAGs)] make the skin and collagenous tissues thick-

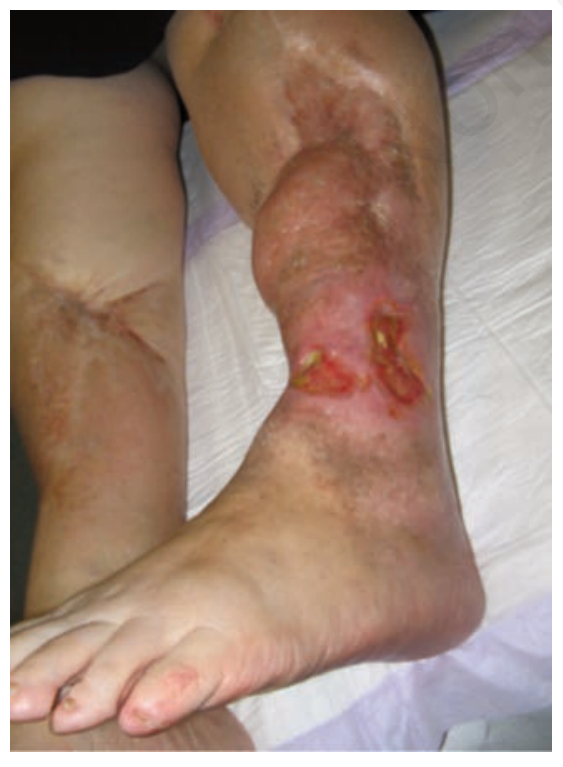

Figure 1. Disfigured and non-homogenous limb following polytrauma: fragility of grafted areas and loss of function.

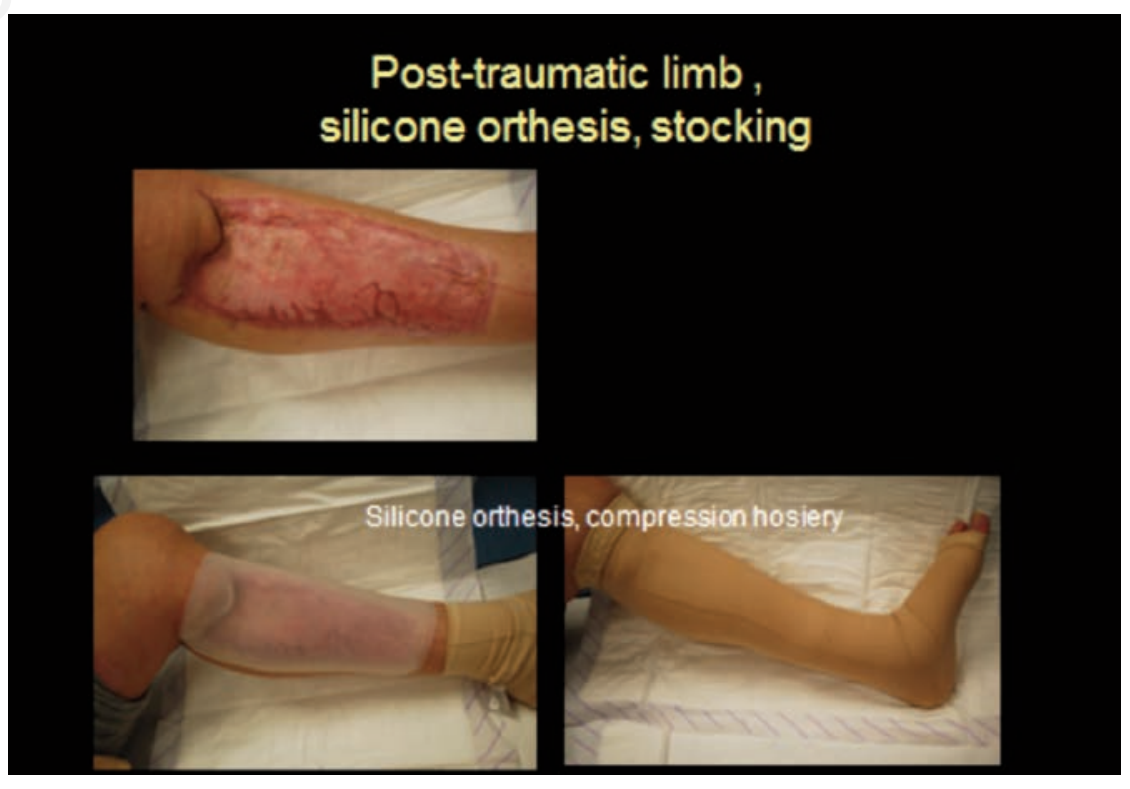

Figure 2. A silicone orthosis might help to reshape the leg in order to permit bandaging or donning of a compression stocking. 

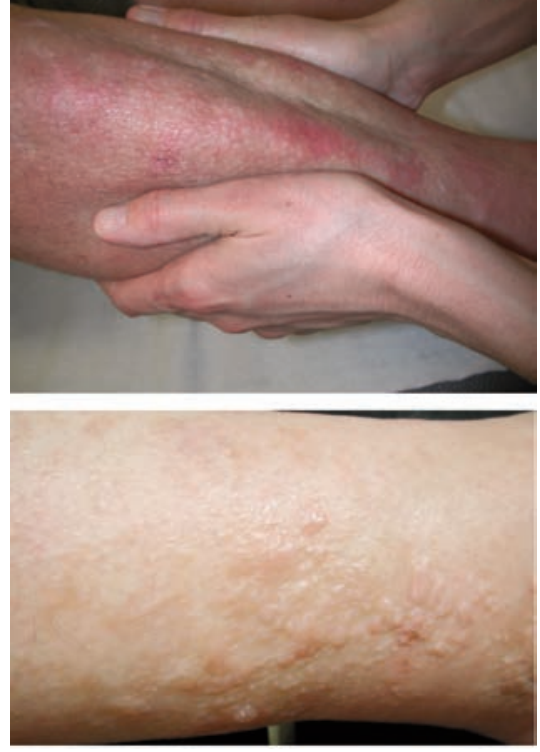

Figure 3. Diabetic thick waxy skin due to deposition of glycosaminoglycans and advanced glycation endproducts.

ened, stiff/less mobile, while at the same time there is retention of fluids leading to edema.

Furthermore, there are advanced fructosylation endproducts, which actually have a greater affinity binding to proteins than glucose and follow a similar pattern in the production of the reactive oxygen species. This huge waxy skin in diabetes not only is at risk for injury leading to blisters or wounds, it is also more prone to inflammation in the context of the dysregulated diabetes (Figure 3). Therefore, compression treatment may be indicated to lower the water content of skin and subcutaneous tissues. Although diabetes is considered as a relative contra-indication for compression treatment of limbs, many have clinical experience of the benefit of compression in these patients and some authors have published beneficial results in diabetic patients without obvious vascular compromise. ${ }^{2}$ In a more recently published study of Wu et al., mild compression therapy $(18-25 \mathrm{mmHg})$ decreased swelling in diabetes patients with lower extremity edema without compromising vascularity. ${ }^{3}$ Interpretation should be cautious considering some weaknesses of this publication: this study is a pilot, uncontrolled study, including a low number of participants. Vascular status was tracked via $\mathrm{ABI}$ (which in diabetics is not always reliable), and edema was measured via tape measurement of circumference at difficult anatomical sites like ankles and mid-foot. The myxedematous skin in thyroid disorders may present specific skin lesions which are due to the storage of large amounts of proteoglycans or mucin deposition in the reticular middle and lower dermis, also responsible for the yellowish color of the skin in these situations. Deposited GAGs
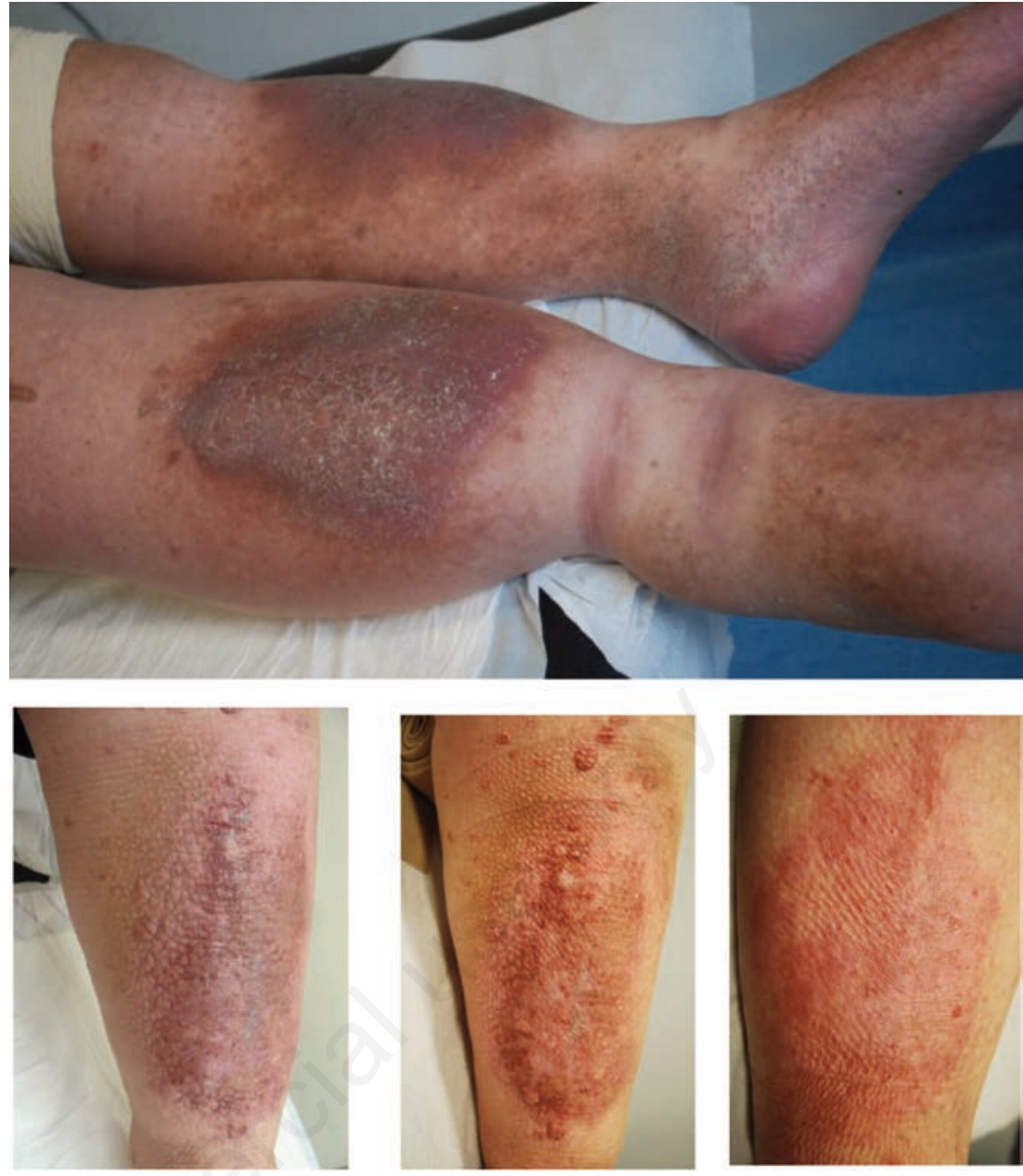

Figure 4. Pretibial myxoedema and effect of compression therapy.

promote dermal edema by promoting the retention of fluid in the skin. The edema is doughy, with a manifest pitting sign. Compression therapy will expel or displace edema fluid and diminish inflammatory erythema, but since the underlying mechanism does not itself respond to pressure, this symptomatic treatment has to be maintained.

In morbid obesity and the frequently associated metabolic syndrome, edema may be attributed to pretibial mucin deposition on the shins, known as pretibial myxedema. ${ }^{4}$ The patients with morbid obesity frequently present with bilateral lower extremity pitting edema (sparing the feet and ankles) of gradual and painless onset. The waxy brownish erythematous plaques are found primarily on the shins, and the semitranslucent papules due to mucin deposition at the superficial papillary dermis and around the vessels may easily develop vesicles when fluid accumulation separates the epidermis from the underlying dermis (Figure 4). One of the many synonyms given to this entity is chronic obesity lymphedematous mucinosis. It has been reported that there is an association between lymphedema in pretibial myxedema and mucin deposition. ${ }^{5}$

\section{References}

1. Zimny S, Dessel F, Ehren M, et al. Early detection of microcirculatory impairment in diabetic patients with foot at risk. Diabetes Care 2001;24:1810-4.

2. Belcaro G, Laurora G, Cesarone MR, et al. Microcirculatory effects of elastic stockings in diabetic microangiopathy: a 24 week study. J Cardiovasc Surg 1993;34: 479-82.

3. Wu SC, Crews RT, Najafi B, et al. Safety and efficacy of mild compression (18-25 $\mathrm{mmHg}$ ) therapy in patients with diabetes and lower extremity oedema. J Diabetes Sci Technol 2012;6:641-7.

4. Tokuda Y, Kawachi S, Murata H, Saida T. Chronic obesity lymphooedematous mucinosis: three cases of pretibial mucinosis in obese patients with pitting oedema. $\mathrm{Br}$ J Dermatol 2006;154:157-61.

5. Bull RH, Coburn PR, Mortimer PS. Pretibial myxooedema: a manifestation of lymphoedema? Lancet 1993;341:403-4. 